\title{
Cecile M. Pickart
}

\section{4-2006}

On April 5 2006, Cecile Pickart, an outstanding biochemist, died following a protracted battle with kidney cancer. She was only fifty-one years old. The biomedical research community has lost a superb scientist, valued colleague and dear friend. A memorial celebrating Cecile's life and scientific contributions is being planned for July 21 this year at the Bloomberg School of Public Health at Johns Hopkins University.

Cecile had been a leading investigator in the ubiquitin field from its infancy and she continued to make some of the most seminal discoveries in the field until her death - a point where the field had matured into an enterprise involving thousands of researchers around the world. After graduating summa cum laude in 1976 with a B.S. degree in biology from Furman University in Greenville, South Carolina, Cecile earned a Ph.D. in biochemistry from Brandeis University. There, she cut her teeth in enzymology under the tutelage of one of the premier enzymologists of the day, William Jencks. In 1982, she left to study with another renowned enzymologist, Irwin Rose, at the Fox Chase Cancer Center in Philadelphia, where she began her studies on ubiquitin.

The covalent attachment of ubiquitin to other proteins had only just been discovered, and few details of the enzymes involved were known at the time. Ubiquitin attachment to cellular proteins is often a signal for degradation of those proteins by a multisubunit protease called the proteasome. The ubiquitin-proteasome system controls an astonishing range of biological processes, and malfunctions of the ubiquitin system are known to underlie a growing list of human diseases.

While a postdoctoral fellow in the Rose laboratory, Cecile discovered that multiple, functionally distinct, ubiquitin-conjugating enzymes (E2s) exist. This was the first hint of the enormous diversity of ubiquitin-system enzymes, which was to be revealed much later through molecular genetic studies. She also performed the first thorough biochemical analysis of deubiquitylating enzymes (DUBs), which remove ubiquitin from proteins and other adducts. Again, the broad significance of these enzymes only emerged many years later. Cecile developed an inhibitor of DUBs, ubiquitin aldehyde, which became a reagent of great practical importance for studying the otherwise highly labile ubiquitin modification in cell lysates.

In 1985, Cecile moved to SUNY, Buffalo, to begin her own laboratory. She initially focused on characterizing the properties of E2 enzymes. Among the E2s that she identified was E2-25K, an enzyme that she, and her graduate student Zhijian (James) Chen, found had the ability to synthesize free polyubiquitin chains, in which the ubiquitins were linked exclusively by amide bonds between Lys 48 of one ubiquitin and the carboxy-terminal carboxyl group of the next. This type of chain, when attached to a substrate protein, had recently been shown by Alex Varshavsky and colleagues at MIT to target the substrate for degradation by the proteasome. The discovery and characterization of E2-25K turned out to be a milestone. Cecile realized that this enzyme, when combined with some elegant chemical tricks, could be used to synthesize polyubiquitin chains of defined length and topology. She used this insight to produce normal and mutant ubiquitin chains in milligram quantities.

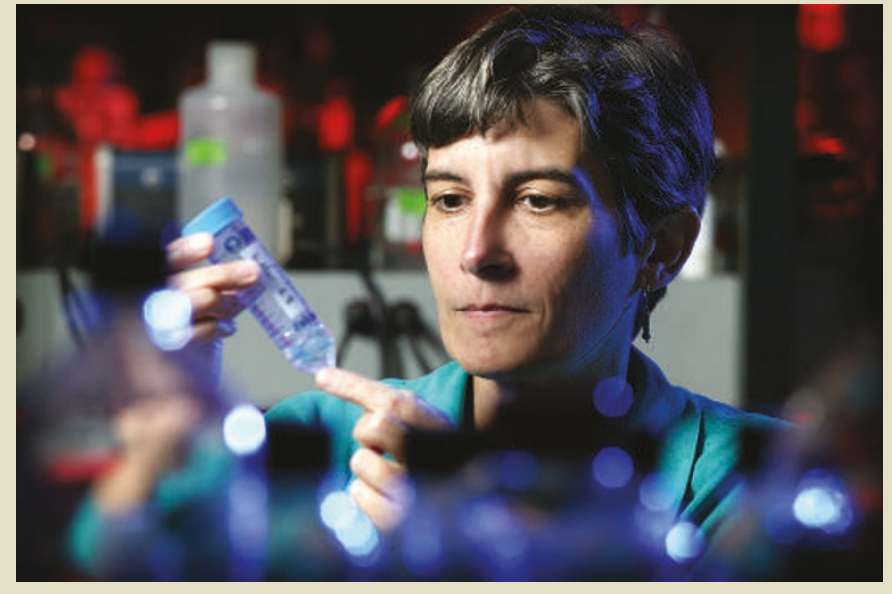

Photo courtesy of the Bloomberg School of Public Health, John Hopkins University

These polyubiquitin chains were used for the first structural studies of ubiquitin polymers, allowing Cecile and her colleagues to identify key residues on the ubiquitin surface that participate in proteasome binding and other ubiquitin functions. She then worked out a method to attach these chains to substrate proteins. This beautiful work culminated in a 2000 paper in the EMBO Journal, where Cecile and colleagues (by then at the Johns Hopkins School of Public Health) demonstrated that the polyubiquitin chain acts to bind substrate proteins to the proteasome. This paper is already a classic.

It soon became apparent that polyubiquitin chains with different ubiquitin-ubiquitin linkages could form in vivo and that these had nonproteolytic functions. Intrigued by this, Cecile purified a protein complex from yeast that was capable of making Lys 63-linked chains. Remarkably, this turned out to be a heterodimer between an E2 and an E2-related protein that was known to function in DNA repair. This discovery, described in a 1999 Cell paper, has led to an avalanche of important findings on Lys 63-linked polyubiquitin chain function and synthesis, many from Cecile's laboratory. Such chains are significant, not only in DNA repair, but for a host of other processes, including signalling by the NF- $\kappa B$ pathway and the endocytosis of membrane proteins.

Cecile brought an uncommon combination of biochemical rigor and creativity to her research. Her laboratory was a constant source of new discoveries with broad impact. She identified the first residue in an E2, besides the thioester-forming cysteine, which participates directly in the catalysis of ubiquitin conjugation. Using difficult chemical crosslinking methods, she identified an ATP-modulated polyubiquitin-binding site in the proteasome. More recently, she began to define how monoubiquitin and different polyubiquitin signals are recognized by ubiquitin-binding proteins. These are questions of central importance to understanding the multitude of ubiquitin functions and underlying mechanisms. A common thread through all of these studies was the unwavering focus on fundamental features of the ubiquitin system.

Along with her stellar research accomplishments, Cecile stood out as both a mentor and a citizen of the scientific community. She committed herself to her students to a degree that few of us can claim. She also helped organize several international meetings on ubiquitin and she served on the NIH biochemistry study section for six years, the last two 
as its chair. Through collaborations, personal contacts, as an anonymous reviewer and as author of many seminal review articles written on different aspects of the ubiquitin system, Cecile helped literally hundreds of scientists negotiate the complexities of the field. Her generosity and energy in these endeavours were extraordinary. Less than two weeks before her death, she was still in the laboratory, purifying enzymes that collaborators and students could then use in their work. In all likelihood, few realized that many key reagents, such as ubiquitin chains and anti-ubiquitin antibodies, that the Pickart laboratory had shared with other groups were actually made by Cecile.

For all her achievements, Cecile's impact was probably not appreciated to the degree it should have been by the broader research community. Although she was elected a Fellow of the American Association for the Advancement of Science and, only this April, to the American Academy of Arts \& Sciences, she had received surprisingly few honours of this type given her extraordinary influence on such an important area of biology. But we in the ubiquitin field know the magnitude of our loss. Cecile's legacy extends well beyond her many scientific breakthroughs. Because she was part of the ubiquitin field from its very beginning and as she was so instrumental in its early development, she also helped shape its 'personality' - the way in which people discuss and share their work, support one another and interact at meetings. Her style was rigorous, honest and deeply decent.

Some years ago, a student who was leaving Cecile's laboratory presented her with a small statue of Athena, the Greek goddess of wisdom. The gift was given in a light-hearted spirit, but it was meant to convey a deep appreciation for the superb mentoring and care given by Cecile during the student's time in her laboratory. The ubiquitin field has lost its Athena. We will all miss the warmth and wisdom of this remarkable woman.

MARK HOCHSTRASSER AND ROBERT COHEN

Mark Hochstrasser is in the Department of Molecular Biophysics \& Biochemistry, Yale University, 266 Whitney Avenue, P.O. Box 208114, New Haven, CT 065208114 , USA. Robert Cohen is in the Johns Hopkins School of Public Health, Department of Biochemistry \& Molecular Biology, 615 North Wolfe Street, Baltimore, MD 21205, USA.

e-mail: mark.hochstrasser@yale.edu; rcohen@jhsph.edu 